\title{
The Dynamics of an Articulated Forestry Machine and its Applications
}

\author{
Evangelos Papadopoulos, Soumen Sarkar
}

\author{
Department of Mechanical Engineering \& Centre for Intelligent Machines \\ McGill University \\ Montreal, PQ, Canada H3A 2A7
}

\begin{abstract}
This paper focuses on the generation of dynamic models for an articulated forestry machine. Such models can be used for training simulators, for sizing components, and for control. The most complex model includes base compliance, and pendulum-like motions of the processing head suspended from an end-point. A Newton-Euler iterative method, implemented symbolically, is used to include base degrees-of-freedom due to the machine's compliant tires. Techniques and experiments designed to extract system parameters are described. Based on the obtained models, a novel valve-sizing methodology is outlined. Finally, simulation results of the machine's response are provided.
\end{abstract}

\section{Introduction}

Forestry is Canada's most important industry in terms of net contribution to its economy [1]. However, competition from overseas and new environmental laws require that forestry resources are harvested more efficiently and more carefully than previously. This requires more sophisticated forestry equipment appropriate to available forests, and that allows for increased harvesting capacity with less damage to the soil and the trees, and for selective logging. Also, such equipment should be easier to control and less tiring, so that operators can focus in planning the operation better.

Many of these requirements can be met by the addition of an on-board information system that can be used for assisting in repetitive tasks, for diagnosing the state of the machine, and for controlling it. The availability of costeffective industrial grade computers, and actuator-sensor mechatronic packages that can withstand the harsh forestry environment, make such "computerization" of forestry machines possible. In fact, some North-American and Scandinavian forestry machines already incorporate some of these systems. For example there exist harvesters which also cut logs to pre specified lengths using opto-electronic or mechanical measurements of log length and diameter.

Many of the existing felling machines are modified construction machines, usually large excavators. Typical modifications include geometrical modifications for better workspace utilization, and addition of specialized processing heads, controlled from the cabin with separate interfaces. Work on coordinated control of excavator-type of machines has began in mid-eighties by P.D. Lawrence and his team [2,3]. In this work, an excavator end-point is controlled in cylindrical task space coordinates by an operator rotating with the arm and using a single joystick. However, an important trend in forestry equipment is designing machines for the environment they work in. Such machines should have the appropriate workspace size and shape, be lightweight, be maneuverable, and agile. In contrast to excavator machines, the operator of a machine designed for forestry operations may be sitting in a nonmoving cabin, and commanding the manipulator in Cartesian space. In addition, actuation systems for such machines are being improved, and are increasingly based on fast closed-center proportional valves, and constant pressure supplies.

This paper reports work focusing on modeling an experimental forestry machine and its applications. Detailed dynamical models at various degrees of complexity are developed to help in designing an effective coordinated controller in Cartesian space, and in developing a training simulator for novice operators. A Newton-Euler iterative method, implemented symbolically, is used to include base degrees-offreedom (dof) due to the machine's compliant tires. The models also include pendulum-type motions of the suspended processing head. These models differ from standard dynamical models developed in robotics primarily because some of the system degrees-of-freedom are either not actuated, or are subject to uncontrolled forces due to base compliance. Parameters for these models were obtained from drawings, actual measurements, simple experiments, and solid modeling techniques. A novel valve sizing methodology is presented based on the dynamic models developed. Finally, simulated response results are presented.

\section{The FERIC Experimental Machine}

The work described here is part of a recent Canadian initiative in forestry robotics, called 'ATREF' (Application des Technologies Robotiques aux Équipements Forestiers) [4]. The machine used was provided by the Forest Engineering Research Institute of Canada (FERIC), and consists of an articulated base that can adjust its pitch by means of a cylinder, and of an articulated manipulator including a mo- 
tor-actuated swing joint, and cylinder-actuated boom and stick joints. An end-point Hooke-type assembly permits free swinging of the processing head in 2 dofs, see Fig. 1.

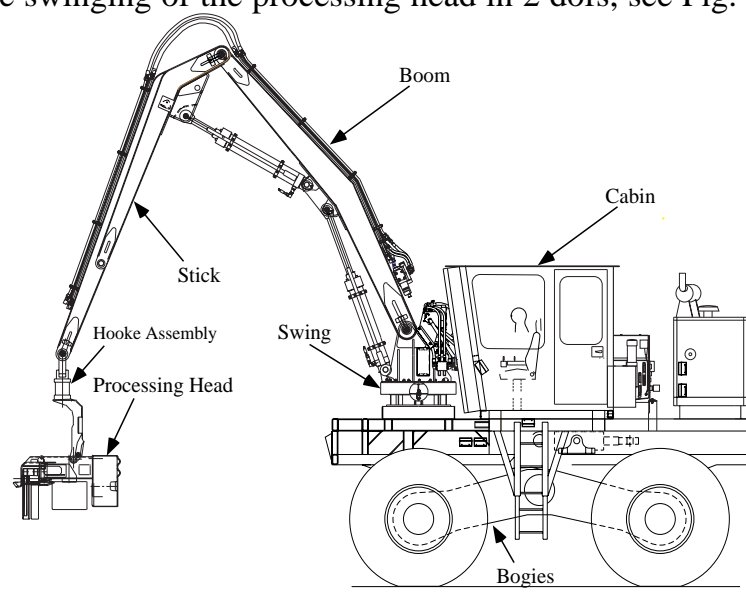

Fig. 1. The FERIC Experimental Machine.

To improve operator visibility, machine stability, and workspace, the original experimental machine, was modified by relocating the cabin and manipulator. Manipulator structural modifications were also necessary to increase the workspace area proximal to the ground. Kinematic modeling of the arm confirmed that lengthening of the stick had the desired effect on its workspace, see Fig. 2.

For the purposes of control and identification experiments, the machine was equipped with magnetostrictive sensors (measuring cylinder displacement), resolvers (measuring angles), inclinometers (measuring vehicle orientation), and flow and pressure sensors for the hydraulics related experiments. More details can be found in Ref. [4].

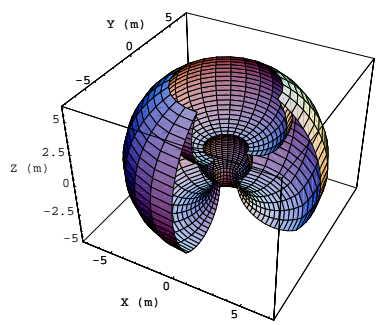

(a)

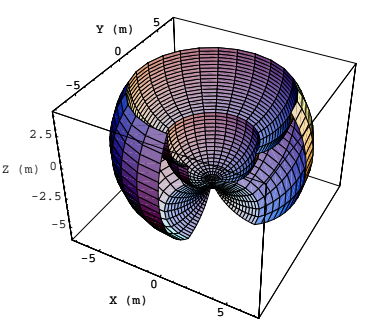

(b)
Fig. 2. The reachable workspace of the manipulator with (a) original stick, (b) extended stick.

\section{Dynamic Modeling}

In contrast to industrial manipulators which are mounted on fixed bases, a forestry manipulator is mounted on a moving and compliant base introducing additional non-actuated dofs. These characteristics introduce additional complexity to the dynamic modelling and control of such systems.

To derive dynamic models of the machine shown in Fig. 1, the iterative Newton-Euler dynamic formulation was chosen because it is easy to implement in the form of computer code, and requires a smaller number of computations $[5,6]$. In this method, kinematic quantities are calculated with outward computations starting from the manipulator base and ending at its tip, while actuator forces and torques are computed with inward computations. A limitation of the numerical iterative Newton-Euler algorithm is that it was developed for fixed-base systems in which all dofs are actuated. In such a case, known desired trajectories for all joints, or dofs, are used to calculate numerically the forces and torques necessary to cause the desired motion. This is not possible in the case of a manipulator mounted on a compliant base, since the base is not actuated, and its position, velocity and acceleration will depend on how fast the arm moves, the load being manipulated, etc. To avoid this problem, it was decided to apply the formulation symbolically, so as to obtain a closed set of symbolic equations of motion. This approach is explained in detail below.

The machine, excluding its manipulator and tires, is modeled as a lumped mass, referred to as the 'base', see Fig. 3.

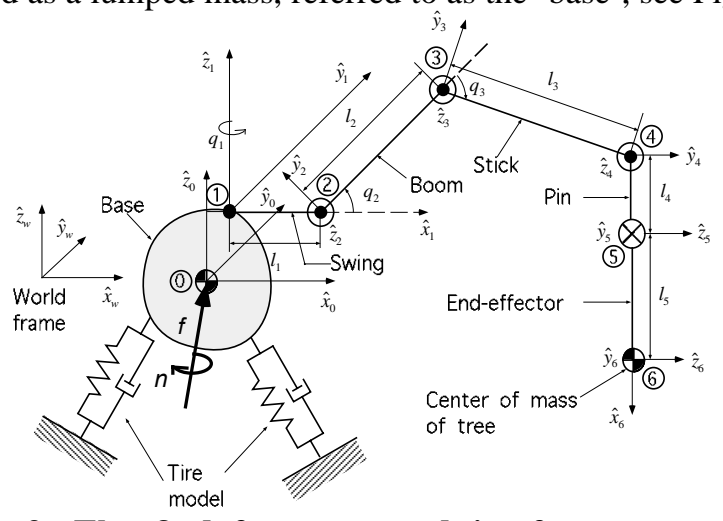

Fig. 3. The 8 dof system and its frames.

A body fixed frame 0 , defined by $\left(\hat{x}_{0}, \hat{y}_{0}, \hat{z}_{0}\right)$, is attached to the center of mass (CM) of the base, and has the same orientation with a world-fixed frame, $\left(\hat{x}_{w}, \hat{y}_{w}, \hat{z}_{w}\right)$, when the vehicle tires are not compressed. The $\hat{x}_{0}$ axis of frame 0 is along the vehicle forward motion, while the $\hat{z}_{0}$ axis is normal to the base and pointing upwards. There are five links in the manipulator namely (from base to tip) the swing, boom, stick, pin and end-effector. The last two links are not actuated but instead they are connected with free joints, subject to frictional effects. A load attached to the last link swings like a double pendulum (gimbals). On all five links, frames are attached as shown in Fig. 3, according to the modified Denavit-Hartenberg methodology, [7]. The swing frame, i.e. frame 1 , has the same orientation with the base frame 0 , when the angle of rotation is zero.

A force/moment set, $(f, n)$, is applied to the base through the tires and the ground. It is reasonable to assume that the soil has been compacted, and that most of the base compliance is due to the machine's pneumatic tires. These four tires are modeled as four springs and dampers in parallel. The simultaneous vertical displacement of the springs gives rise to a base bouncing effect. Due to the spring structure, the base is also subject to pitch (rotation around the $\hat{y}_{0}$ axis) and roll (rotation around the $\hat{x}_{0}$ axis) motions. 
The yaw effects as well as displacements along the $\hat{x}_{w}$ and $\hat{y}_{w}$ axes are usually negligible, and therefore are neglected.

To apply the Newton-Euler formulation, special care must be placed in including base compliance. To obtain the equations, it is assumed that the position, velocity, and acceleration of the base is known. Assuming that the motion of the links is also known, allows finding all link accelerations, and from these all body forces and moments. Once an expression for the forces and moments acting on the base is found, it is equated to the spring and damper forces due to the tires, which are a function of base position and velocity. This closes the equations of motions, which are then written in the standard matrix form and integrated.

In more detail, the base orientation is described by two successive Euler transformations (yaw neglected)

$$
{ }^{w} R_{0}=\left[\begin{array}{ccc}
c_{y} & 0 & s_{y} \\
0 & 1 & 0 \\
-s_{y} & 0 & c_{y}
\end{array}\right]\left[\begin{array}{ccc}
1 & 0 & 0 \\
0 & c_{x} & -s_{x} \\
0 & s_{x} & c_{x}
\end{array}\right]
$$

where $c_{z}$ is the cosine of $q_{z}$ and $s_{z}$ is the sine of $q_{z}$, etc. The angles $q_{x}$, and $q_{y}$, are the roll, and pitch respectively.

The position of the base frame with respect to the world frame is defined as

$$
{ }^{w} p_{0}^{w}=\left[\begin{array}{lll}
x & y & z
\end{array}\right]^{T},
$$

where the symbol ${ }^{a} p_{c}^{b}$ should be read as the position vector of point $c$ with respect to point $b$ expressed in frame $a$. To start the kinematic propagation equations, we set

$$
{ }^{w} v_{w}=\left[\begin{array}{l}
0 \\
0 \\
0
\end{array}\right],{ }^{w} \dot{v}_{w}=\left[\begin{array}{l}
0 \\
0 \\
g
\end{array}\right],{ }^{w} \omega_{w}=\left[\begin{array}{l}
0 \\
0 \\
0
\end{array}\right],{ }^{w} \dot{\omega}_{w}=\left[\begin{array}{l}
0 \\
0 \\
0
\end{array}\right]
$$

Note that to model a sloped terrain, it suffices to modify the components of ${ }^{w} \dot{v}_{w}$. Applying propagation equations, we obtain for the base

$$
{ }^{0} \omega_{0}={ }^{0} R_{w}{ }^{w} \omega_{w}+\left[\begin{array}{c}
\dot{q}_{x} \\
c_{x} \dot{q}_{y} \\
-s_{y} \dot{q}_{y}
\end{array}\right]=\left[\begin{array}{c}
\dot{q}_{x} \\
c_{x} \dot{q}_{y} \\
-s_{y} \dot{q}_{y}
\end{array}\right]
$$

Only the vertical displacement is considered, therefore,

$$
\begin{gathered}
{ }^{0} v_{0}={ }^{0} R_{w}\left({ }^{w} v_{w}+{ }^{w} \omega_{w}{ }^{\times w} p_{0}^{w}\right)+\left[\begin{array}{l}
0 \\
0 \\
\dot{z}
\end{array}\right]=\left[\begin{array}{l}
0 \\
0 \\
\dot{z}
\end{array}\right] \\
{ }^{0} \dot{v}_{0}={ }^{0} R_{w}\left[{ }^{w} \dot{v}_{w}+{ }^{w} \dot{\omega}_{w} \times{ }^{w} p_{0}^{w}+{ }^{w} \omega_{w} \times\left({ }^{w} \omega_{w} \times{ }^{\times w} p_{0}^{w}\right)\right] \\
+2\left({ }^{0} R_{w}{ }^{w} \omega_{w}\right)^{\times}\left[\begin{array}{l}
0 \\
0 \\
\dot{z}
\end{array}\right]+\left[\begin{array}{l}
0 \\
0 \\
\ddot{z}
\end{array}\right] \\
{ }^{0} \dot{v}_{c_{0}}={ }^{0} \dot{v}_{0}+{ }^{0} \dot{\omega}_{0}^{\times 0} p_{c_{0}}^{0}+{ }^{0} \omega_{0} \times\left({ }^{0} \omega_{0} \times{ }^{\times 0} p_{c_{0}}^{0}\right)={ }^{0} \dot{v}_{0}
\end{gathered}
$$

where ${ }^{0} p_{c_{0}}^{0}=0$, and $c_{0}$ indicate the $\mathrm{CM}$ of the base. The total force and moment acting on the base are given by

$$
{ }^{0} F_{0}=m_{0}{ }^{0} \dot{v}_{c_{0}},{ }^{0} N_{0}={ }^{0} I_{0}^{c 0} \dot{\omega}_{0}+{ }^{0} \omega_{0} \times{ }^{0} I_{0}^{c 0} \omega_{0}
$$

where the subscript ${ }^{\times}$converts a vector to a skew-symmetric cross-product matrix, $m_{0}$ and ${ }^{0} \dot{v}_{c_{0}}$ are the mass of the base and acceleration of the center of mass of the base expressed in base frame $0,{ }^{i} I_{i}^{c}$ is the inertia tensor of the $i$ th link, and ${ }^{i} F_{i}$ and ${ }^{i} N_{i}$ denote the force and moment acting at the center of mass of the $i$ th link expressed in the $i$ th frame.

Once the base velocities and accelerations are found, then the procedure continues by forward iterations from link 1 to 5, till the end-point is reached. During the backward iteration, constraint forces and torques, and joint actuator torques are computed recursively from link 5 to link 1 . A more detailed description of this procedure can be found in [7]. The forces and torques transmitted to the base by the tires can then be found from the last inward iteration using the following equations

$$
{ }^{0} f_{0}={ }^{0} R_{1}{ }^{1} f_{1}+{ }^{0} F_{0},{ }^{0} n_{0}={ }^{0} N_{0}+{ }^{0} R_{1}{ }^{1} n_{1}+{ }^{0} p_{c_{0}}^{0 \times 0} F_{0}+{ }^{0} p_{1}^{0 \times 0} R_{1}{ }^{1} f_{1}
$$

where the symbols ${ }^{i} f_{i}$ and ${ }^{i} n_{i}$ denote the force and torque exerted on link $i$ by link $i-1$, expressed in frame $i$. These can be expressed in world frame as

$$
{ }^{w} f_{0}={ }^{w} R_{0}{ }^{0} f_{0}, \quad{ }^{w} n_{0}={ }^{w} R_{0}{ }^{0} n_{0}
$$

where ${ }^{w} R_{0}$ is given by Eq. (1). Since the tires are modeled as springs and dampers, then

$$
F=\left[\begin{array}{ll}
{ }^{w} f_{0} & { }^{w} n_{0}
\end{array}\right]^{T}=\left[\begin{array}{ll}
f & n
\end{array}\right]^{T}=-K X-B \dot{X}
$$

where $X$ and $\dot{X}$ are the linear and angular displacement and velocity vectors of the base with respect to world frame. $K$ and $B$ are the stiffness and damping matrices and capture the effect of tire behavior. For simplicity, and for small motions, these matrices are assumed to be diagonal

$$
K=\operatorname{diag}\left(k_{x}, k_{y}, k_{z}, k_{1}, k_{2}, k_{3}\right) B=\operatorname{diag}\left(b_{x}, b_{y}, b_{z}, b_{1}, b_{2}, b_{3}\right)
$$

The symbols $k_{x}, k_{y}$ and $k_{z}$ represent the total linear stiffnesses along the corresponding directions, as denoted by subscript with respect to the world frame. The term 'total' stiffness is used to represent the combined stiffness of the four tires. The $k_{1}, k_{2}$ and $k_{3}$ represent the total angular stiffness for roll, pitch and yaw. The same notation is applied in the case of damping. Since only three base motions are significant, i.e., bounce, roll and pitch, the remaining base equations are dropped. Then, the other two displacements are constant and the yaw angle is zero.

Finally, the equations of motion are written as

$$
M \ddot{q}+V(q, \dot{q})+G(q)=Q
$$

where $M$ is an $8 \times 8$ symmetric and positive definite mass matrix, $V$ contains the Coriolis and centrifugal terms, $G$ the gravity terms, and $Q$ is the force/torque vector. A reduced five dof order model is formed by neglecting base compliance in the eight dof model. This model will be used as the dynamics engine of a real-time training simulator also undergoing development as part of the ATREF project [8]. A further reduced model of three dofs, formed by 
neglecting the Hooke-type gimbals, has been used for the valve sizing studies, described in Section 5.

\section{Parameter Estimation}

Model parameters are needed to run the simulations, validate the developed code, and design controllers. Geometrical parameters such as lengths were found from blueprints, and verified by direct measurements. Some masses were computed using drawings, or by direct weighing. For the rest of the parameters, a combination of pendulum experiments and solid modeling techniques was used. The stiffness and damping ratio of the tires were found by static load-deflection tests and drop tests, respectively.

\section{4a. Pendulum Experiments}

During a pendulum experiment, a rigid body is suspended from a point, is angularly displaced, and then is set free to swing. The period of the resulting oscillation is recorded, and is subsequently used to calculate the moment of inertia around the axis of rotation according to

$$
I_{z z}^{0}=\frac{m g l T^{2}}{4 \pi^{2}}
$$

where $I_{z z}^{0}$ is the moment of inertia of the body with respect to the axis of swinging (a $z$-axis), $m$ is its mass, $T$ is the period of oscillation, and $l$ is the length from the point of suspension to body CM. The moments of inertia with respect to body CM, are then computed using the parallel axis theorem. As revealed by Eq. (14), the inertia is proportional to the square of the time period, and this may result in substantial estimation errors. Moreover, swinging a body with respect to a single axis is a difficult task. For these reasons, pendulum experiments were used in parallel to solid modeling techniques.

\section{4b. Solid Modeling}

Solid modeling techniques can be used in obtaining all mass properties and center-of-mass positions, assuming that the material and the geometry of a body or link are precisely known. However, this is not always the case. To match solid modeling estimates to measurements, static suspension, weighing, and pendulum experiments were used to refine solid models to the point that both the estimated and measured total mass and moment of inertia were in agreement. The solid models generated for the swing and the boom are shown in Fig. 4. Following the techniques described above, the inertia parameters of the main links were obtained and are given in Table I.

\section{4c. Load-deflection Experiment}

To obtain the tire stiffness, $k$, load-deflection experiments were conducted. In these, a load is applied on a tire and its vertical deflection is measured. Fig. 5a shows a typical plot obtained from such experiment. As shown by this figure, the tire behaves like a linear spring in the region of loads of interest. From the average slope of the plot in Fig. 5a, the tire stiffness was computed as equal to $k=49.23 \mathrm{~kg} / \mathrm{mm}$. This stiffness was used to calculate the translational and angular stiffness for bounce, roll and pitch.
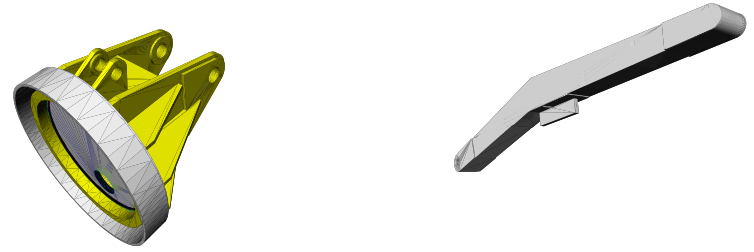

Fig. 4. Solid models generated in AutoCad.

Table I. Link Inertia Properties.

\begin{tabular}{|c|c|c|c|c|c|c|}
\hline in $\mathrm{kgm}^{2}$ & $I_{x x}$ & $I_{y y}$ & $I_{z z}$ & $I_{x y}$ & $I_{y z}$ & $I_{z x}$ \\
\hline Swing & 52 & 53 & 56 & .01 & .02 & 5 \\
\hline Boom & 17 & 926 & 929 & .36 & -.09 & -.70 \\
\hline Stick & 16 & 816 & 826 & 32 & .14 & .51 \\
\hline Pin & 0 & 3.3 & 3.3 & 0 & 0 & 0 \\
\hline End-eff. & 0 & 1265 & 1265 & 0 & 0 & 0 \\
\hline
\end{tabular}

\section{4d. Drop Experiment}

One of the simplest methods to estimate the damping ratio of a non-rolling tire is the so-called drop test [9]. In the case of a light tire, a load is added to the hub of the tire, which is just in contact with a steel slab, without deforming it (the load is supported externally). The load is then set free, and the loaded tire is allowed to deform freely from its initial position. Throughout the experiment, the tire must be in contact with the slab, otherwise obtained results will not be valid due to impacts. An accelerometer mounted on its hub records the tire transient response, which corresponds to an underdamped oscillation. Fig. 5b displays a typical accelerometer reading during a drop experiment.

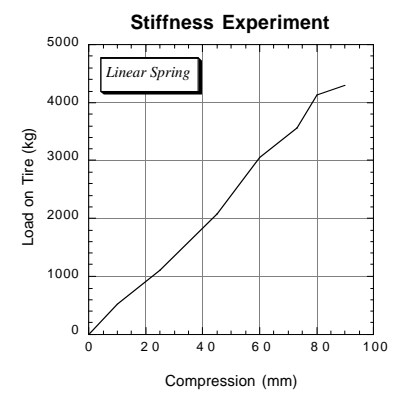

(a)

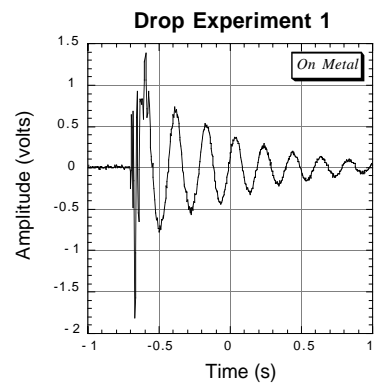

(b)
Fig. 5. (a) Tire stiffness estimation, (b) Tire damping estimation.

Double integration of the acceleration data such as the one displayed in Fig. 5b, yields the hub displacement response. Then, using the amplitude of two successive periods of the response, $x_{1}$ and $x_{2}$ and the logarithmic decrement equation, the damping ratio coefficient, $\zeta$, is found according to 


$$
\ln \left(\frac{x_{1}}{x_{2}}\right)=\frac{2 \pi \zeta}{\sqrt{1-\zeta^{2}}}
$$

Using this equation, the value of the damping ratio was computed as 0.035 . Based on this estimate, and on the mass of the tire, the tire damping coefficient is calculated. The damping coefficient for the pitch, roll, and bounce can then be computed easily [6].

\section{Valve Sizing Based on Inverse Dynamics}

An important application of dynamic modeling is sizing of actuators. In the case of the experimental electrohydraulic machine, it has been decided not to replace the existing hydraulic cylinders or the swing motor. However, the need to replace the old load-sensing valves by new proportional ones for the constant-pressure supply, provided an important application for the derived dynamic models. According to typical industrial practice, proportional valves are selected based on a nominal load and duty cycle. However, no such nominal quantities exist for a manipulator arm whose configuration changes continuously, and may carry no load, or be loaded with a heavy tree. Therefore, a systematic methodology for valve sizing was needed.

A valve is properly sized when it can supply the demanded flow at the required pressure drop across it. Therefore to size a valve, flow and pressure requirements must be obtained as a function of time for a given task. Obviously, the task becomes more demanding when the manipulator is moving a heavy payload, or when it operates on a slope. To this end, typical average as well as worstcase trajectories of the manipulator end-point were specified by observation of actual forestry machines. Using inverse kinematics relationships, these end-point trajectories were resolved at the actuator level, to result in trajectories for the swing angle, and the boom and stick displacements. Then, these were used to obtain the flow requirements for all three actuated dofs.

Assuming average piston areas, the flow through the stick and boom valve and cylinder is

$$
Q_{s}=A_{s} \dot{x}_{s}, \quad Q_{b}=A_{b} \dot{x}_{b}
$$

where $A$ is piston area, and $\dot{x}$ is piston velocity. Since the swing is driven by a gearbox motor, the flow through the swing motor valve is

$$
Q_{s w m}=D n \dot{q}_{1}
$$

where $D$ is the motor displacement, $n$ is the gear ratio from the swing link to swing motor, and $\dot{q}_{1}$ is the angular velocity of the manipulator swing link.

To obtain the pressure drops across the three valves, a reduced three dof dynamic model that includes the actuated dofs (swing, boom, stick) was used. From this model and the desired trajectories, the necessary forces at the two cylinders, and the torque necessary to rotate the manipulator were computed using inverse dynamics equations in the form of Eq. (13). These forces and torques are related to the pressure drops $\Delta p$ at the cylinders and the swing motor according to the following equations

$$
\begin{gathered}
\Delta p_{s}=\frac{f_{s}}{A_{s}}, \Delta p_{b}=\frac{f_{b}}{A_{b}} \\
\Delta p_{s w m}=\frac{\tau_{s w m}}{D}=\frac{\tau_{s w}}{n D}
\end{gathered}
$$

where $f_{\mathrm{s}}$ is the force applied by the stick cylinder, $f_{\mathrm{b}}$ is the force applied by the boom cylinder, and $\tau_{\text {swm }}$ is the torque applied by the hydraulic motor. Neglecting line pressure drops, the drop at the valves, $\Delta p_{v}$, are given by

$$
\begin{gathered}
\Delta p_{v, s}=p_{o p}-\left|\Delta p_{s}\right|, \Delta p_{v, b}=p_{o p}-\left|\Delta p_{b}\right| \\
\Delta p_{v, s w m}=p_{o p}-\left|\Delta p_{s w m}\right|
\end{gathered}
$$

where $p_{o p}$ is the constant operating pressure of the machine's pumps. If necessary, these estimates can be decreased by a factor of $10 \%$ to allow for pressure drops in the transmission lines. Equations (16-17) and (19) can be used to plot valve flow versus valve drop for the desired endpoint trajectories. The resulting $Q-\Delta p$ curve should lie below the valve pressure-flow characteristic, $Q_{v}-\Delta p_{v}$, typically a curve described by a relationship of the form,

$$
Q_{v}=c \sqrt{\Delta p_{v}}
$$

If this is not the case, a valve of larger capacity must be specified. Fig. 6 shows typical plots of such curves for the boom, stick, and swing, when the macine is operating on a $20^{\circ}$ sloped terrain. Since all plots lie under the valve characteristic, this valve can be used for driving all manipulator actuators along the desired trajectory.

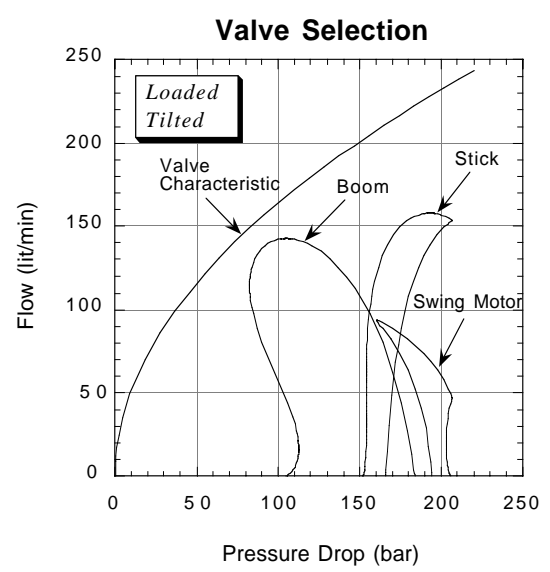

Fig. 6. Boom, stick and swing pressure-flow plots lie below the valve characteristic.

\section{Dynamic Response}

In this section the dynamic behavior of the eight dof system is studied based on torque/force inputs generated by a setpoint feedforward controller [6]. The focus here is to study system transient and steady state response for various joint torques. Figs. 7a,b,c show typical actuator applied torques, while Figs. $7 d$,e,f depict base pitch, roll and bounce. Although these are relatively small, their effect at the end- 
point is not negligible. Finally, the response of the Hooke assembly joint angles is shown in Fig. $7 \mathrm{~g}$, $\mathrm{h}$.

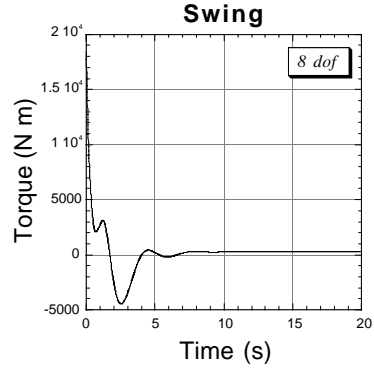

(a)

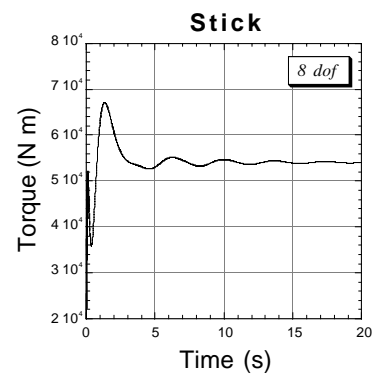

(c)

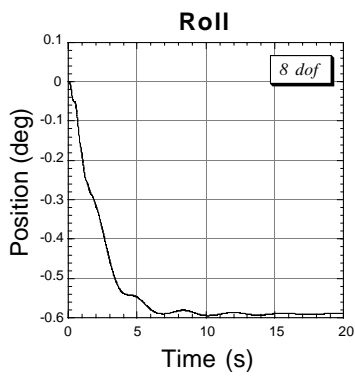

(e)

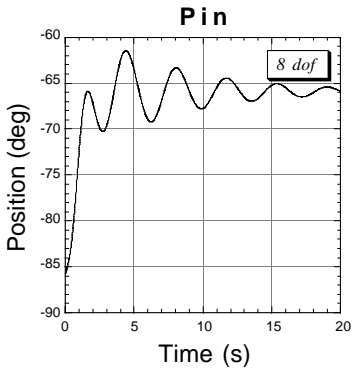

(g)

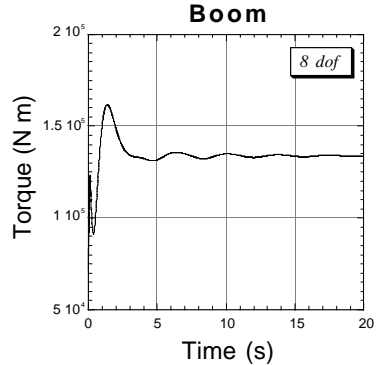

(b)

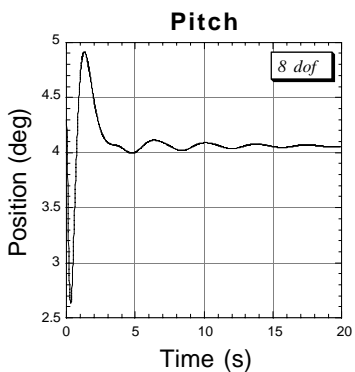

(d)

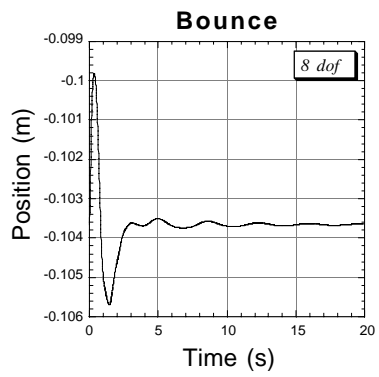

(f)

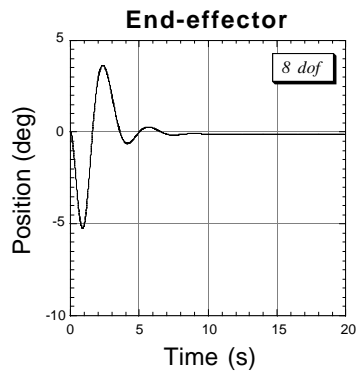

(h)
Fig. 7. Transient response results.

Since these links are not actuated, their response is oscillatory. However, eventually this oscillation dies out due to the presence of joint friction.

\section{Conclusions}

This paper studied the generation of dynamic models for an electrohydraulic forestry machine. Such models can be used for training simulators, for sizing components, and for system design. The most complex model, includes base compliance, and swing, boom, stick, and non-actuated dofs of the processing head, suspended from the end-point. A symbolic version of the Newton-Euler iterative method was used to include the base dofs due to the compliant tires. Techniques and experiments designed to extract system parameters were described. Based on the obtained models, a novel valve-sizing methodology was outlined. Simulation results of the system's response were provided.

\section{Acknowledgments}

The financial support by the Ministère de l'Industrie, du Commerce, de la Science et de la Technologie of Quebec, under the program SYNERGIE is gratefully acknowledged.

\section{References}

[1] Courteau, J., "Robotics in Canadian Forestry," IEEE Canadian Review, Winter 1994, pp. 10-13.

[2] Lawrence, P.D. et al, "Computer-Assisted Control of Excavator-Based Machines," SAE Technical Paper \# 932486, Warrendale, PA, 1993.

[3] Sepehri, N. et al., "Cascade Control of Hydraulically Actuated Manipulators," Robotica, v.8, 1990, pp. 207-216.

[4] Freedman, P., Papadopoulos, E., Poussart, D., Gosselin, C., and Courteau, J, “ATREF: Application des Technologies Robotiques aux Équipements Forestiers," Proc. 1995 Canadian Conf. on Electrical and Computer Engineering, Montreal, PQ, Sept. 5-8, 1995.

[5] Luh, J. Y. S., Walker, M., and Paul, R. P., “OnLine Computational Scheme for Mechanical Manipulators," J. of Dynamic Systems, Measu-rement and Control, Vol. 102, 1980, pp. 69-76.

[6] Sarkar, S., "Dynamic Modeling of an Articulated Forestry Machine for Simulation and Control," Master's Thesis, McGill University, 1996.

[7] Craig, J. J., Introduction to Robotics, Second Edition, Addison-Wesley, Reading, MA, 1989.

[8] Girard, I and Freedman, P., "A Computerized Training Environment for Forestry Operators," Proc. Robotics and Knowledge-based Systems Workshop, Department of National Defense and the Canadian Space Agency, St. Hubert, Que., Oct. 15-18, 1995.

[9] Wong, J. Y., Theory of Ground Vehicles, John Wiley and Sons, Inc., New York, NY, 1993. 